\title{
Acute Disseminated Encephalomyelitis With Brain Abscess in a 3-Year-Old Immunocompetent Child: A Case Report
}

\author{
Pamela Cherfan ${ }^{\mathrm{a}, \mathrm{c}}$, Marianne Touma Boulos ${ }^{\mathrm{a}}$, Doris Elia ${ }^{\mathrm{b}}$, \\ Caroline Geagea ${ }^{a}$, Patricia Kaldany ${ }^{\mathrm{a}}$
}

\begin{abstract}
Acute disseminated encephalomyelitis (ADEM) is a monophasic immune-mediated inflammatory demyelinating condition that predominately affects the white matter of the brain and spinal cord. On magnetic resonance imaging (MRI), lesions are usually diffuse, poorly demarcated, and demonstrate regions of high signal on T2 with surrounding edema. On the other hand, intracranial abscesses are uncommon, serious and life-threatening infections. On MRI, restricted water diffusion, in ring-enhancing lesions, is typical of brain abscesses. We report a challenging case of a 3-year-old boy presenting with fever, severe headache, somnolence and neck stiffness. The cerebrospinal fluid (CSF) showed high white blood cell count and the brain MRI showed two lesions evoking abscesses with multiple areas of vasogenic edema. Six days later and due to the persistence of the somnolence with a gait disturbance despite antibiotic therapy, a follow-up MRI showed a better delineation of the vasogenic edema that now evokes ADEM lesions with regression of the two lesions evoking abscesses. Diagnostic considerations were brainstem abscesses or ADEM or an association between them. However, no association between these two entities has been reported in the literature. Because first MRI lesions were typical of abscesses, the diagnosis of an association between brain abscesses and ADEM was retained. The patient was treated with high doses of steroids that drastically and promptly improved his condition and the antibiotic therapy was continued for 4 weeks despite the absence of bacterial growth on CSF culture. In conclusion, we report here a first case of an association between brain abscesses and ADEM in a child with a good evolution after treatment. The differential diagnosis could be atypical MRI images of ADEM that resembled abscesses.
\end{abstract}

Keywords: Acute disseminated encephalomyelitis; Demyelinating

Manuscript submitted July 15, 2019, accepted August 3, 2019

aDepartment of Pediatrics, Bellevue Medical Center, Saint Joseph University, Beirut, Lebanon

bepartment of Radiology, Bellevue Medical Center, Saint Joseph University, Beirut, Lebanon

${ }^{\mathrm{c} C}$ Corresponding Author: Pamela Cherfan, Department of Pediatrics, Bellevue Medical Center, Beirut 2038 3054, Lebanon.

Email: pamelacherfan@gmail.com

doi: https://doi.org/10.14740/ijcp339 lesion; Brain abscess; Encephalitis; Meningitis; Children

\section{Introduction}

Acute disseminated encephalomyelitis (ADEM) is a monophasic immune-mediated inflammatory demyelinating condition that predominately affects the white matter of the brain and spinal cord [1]. The disorder manifests as an acute onset encephalopathy associated with polyfocal neurologic deficits. ADEM typically follows a febrile infection or vaccination [25]. Cerebrospinal fluid (CSF) analysis may reveal mild pleocytosis and elevated protein, but is generally negative for intrathecal oligoclonal immunoglobulin G synthesis [6]. Magnetic resonance imaging (MRI) is far more sensitive than computed tomography (CT) for the diagnosis of ADEM. Lesions are usually diffuse, poorly demarcated and demonstrate on $\mathrm{T} 2$, regions of high signal with surrounding edema, typically situated in subcortical locations; the thalami and brainstem can also be involved. The center of the lesions does not show increased restriction on diffusion weighted MRI (DWI) [6-8].

On the other hand, intracranial abscesses are uncommon, serious, life-threatening infections. Intracranial abscesses can originate from infection of contiguous structures or can be secondary to hematogenous spread from a remote site or after a skull trauma or surgery, rarely following meningitis. In at least $15 \%$ of cases, no source can be identified [9]. On MRI, restricted water diffusion, in ring-enhancing lesions, is typical of brain abscess [10].

We report a challenging case of a 3-year-old boy presenting with fever, severe headache, somnolence and neck stiffness. Images on MRI showed lesions evoking abscesses and others evoking ADEM. Differential diagnoses were brainstem abscesses or ADEM or an association between them. A single diagnosis could not be retained due to the different types of lesions on the MRI. However, no association between these two entities has been reported in the literature.

\section{Case Report}

A 3-year-old boy presented to the emergency room with a 




Figure 1. MRI showing vasogenic edema contiguous to the third ventricle centered by diffusion restriction (thin arrow) and annular post contrast enhancement (thick arrow). (a) FLAIR coronal sequence, (b) T1-weighted axial sequence, and (c) diffusion weighted imaging axial sequence. MRI: magnetic resonance imaging; FLAIR: fluid attenuation inversion recovery.

4-day history of high grade fever $\left(>39^{\circ} \mathrm{C}\right)$, mild headache, lethargy and somnolence associated with dry cough and left eye redness. He is a healthy young boy coming from a middle class family. His parents are not related and he has a healthy 1-year-old younger brother. He had an appropriate cognitive behavior and a good growth (75th percentile for height and weight). He received all the required vaccines for his age: four doses of diphtheria tetatnus pertussis polio hemophilus influenza B vaccine, three doses of hepatitis B vaccine, four doses of pneumococcal conjugate vaccine 13 , one dose of meningococcal groups $\mathrm{A}, \mathrm{C}, \mathrm{Y}$ and $\mathrm{W}-135$ polysaccaride diphtheria toxoid conjugate vaccine at 2 years of age, two doses of measles mumps and rubella vaccine, two doses of hepatitis A vaccine and one dose of typhoid vaccine. He did not receive any vaccination during the last 3 months. He attended daycare since the age of 2 years and has a history of recurrent otitis media for which he was operated 3 months ago of myringotomy and titanium tube placement.

Two weeks prior to his admission to the emergency room (ER), he had a 3-day history of mild fever with runny nose that resolved spontaneously. Upon admission, the patient was febrile but hemodynamically stable. His Glasgow coma score was $14 / 15$. The physical exam showed minimal neck stiffness and mild somnolence, otherwise normal. Initial blood tests revealed hematocrit of $34 \%$, white blood cell (WBC) count of $13,700 / \mathrm{mm}^{3}$ with relative neutrophilia $(63 \%)$, platelet count of $369,000 / \mathrm{mm}^{3}$ and C-reactive protein (CRP) of $80 \mathrm{mg} / \mathrm{dL}$. No electrolytes disturbances were noted. CSF from the lumbar puncture showed elevated WBC count of $308 / \mathrm{mm}^{3}$ (54\% neutrophils, $40 \%$ lymphocytes). The glucose in the CSF was 57 $\mathrm{mg} / \mathrm{dL}$ (blood glucose was $86 \mathrm{mg} / \mathrm{dL}$ ), and the protein in CSF was $0.2 \mathrm{~g} / \mathrm{L}$. No microorganism was seen on gram stain and bacterial antigen detection test on CSF was negative.

Meningitis was suspected in front of the clinical picture and the presence of high WBC in the CSF and patient was started on intravenous (IV) antibiotics (ceftriaxone $100 \mathrm{mg} / \mathrm{kg} /$ day, vancomycin $80 \mathrm{mg} / \mathrm{kg}$ and amikacin $15 \mathrm{mg} / \mathrm{kg} / \mathrm{day}$ ). Cultures from CSF, blood and urine were taken before starting the antibiotics, as well as viral polymerase chain reaction (PCR) panel on CSF (herpes simplex viruses 1 and 2, varicella-zoster virus, Ebstein-Bar virus (EBV), cytomegalovirus (CMV), herpes simplex viruses 6,7 and 8, enterovirus).

Twenty-four hours later, the patient was still febrile and somnolent with an unsteady gait. Acyclovir $(60 \mathrm{mg} / \mathrm{kg} / \mathrm{day})$ was added to the antibiotics to cover possible herpes encephalitis, and a brain MRI was done and showed multiple areas of vasogenic edema contiguous to the third ventricle and at the level of the brainstem. These areas were centered by two foci of diffusion restriction and annular post contrast enhancement. Note was made for mild posterior fossa meningeal enhancement (Fig. 1). Because of the annular enhancing lesions and the mild posterior fossa meningeal enhancement, brain abscesses were the most plausible diagnosis. Metronidazole at a dose of $35 \mathrm{mg} / \mathrm{kg} /$ day was added to the previously given antibiotics. He also received a dose of dexamethasone $(0.6 \mathrm{mg} / \mathrm{kg} /$ day) to decrease the brain edema. Acyclovir was discontinued after 4 days because CSF viral PCR result was negative. CSF, blood and urine cultures taken on admission were negative for bacterial growth. A CT scan of paranasal sinuses searching for a source of infection showed a subacute sinusitis.

Clinically the patient was afebrile $24 \mathrm{~h}$ after the initiation of metronidazole but the headache and the intermittent somnolence were persistent.

On day 6 of admission ( 5 days after the first MRI), and because of this persisting somnolence and unsteady gait, a follow-up brain MRI was done and showed a mild regression of the previously described foci of diffusion restriction and annular enhancement with better delineation of the vasogenic edema lesions in the posterior fossa without evidence of newly appearing hyperintense $\mathrm{T} 2$ fluid attenuation inversion recovery (FLAIR) lesions. The meningeal enhancement described previously was no longer seen. The follow-up lumbar puncture was also done on day 6 of admission, showing a clear CSF, a decrease in WBC count to $25 / \mathrm{mm}^{3}$, no oligoclonal bands, no organism seen on gram stain, no bacterial growth in CSF culture and a negative viral PCR in CSF. Blood cultures and blood serology for EBV, CMV and toxoplasmosis were negative as well as aquaporin-4 antibodies for neuromyelitis optica.

Based on the radiologic evolution, and because the patient was still somnolent with an unsteady gait, the diagnosis of 
ADEM was considered. The child was started on high-dose IV methylprednisolone $(30 \mathrm{mg} / \mathrm{kg} /$ day $)$. He improved drastically $24 \mathrm{~h}$ after receiving the first dose of methylprednisolone. He received a 3-day course of high-dose methylprednisolone followed by a tapering of the dose over 10 days.

The MRI images were seen by one pediatric radiologist and two neuro-pediatric radiologists and because the presence of an abscess could not be ruled out due to the presence of diffusion restriction at the level of the sub-thalamic area and the post contrast annular enhancement on the initial MRI study, the IV antibiotics (vancomycin, metronidazole and ceftriaxone) were continued for a total of 4 weeks, although no bacterial growth was noted on both CSF taken (at day 1 and at day 6) and although the clinical evolution was suggestive of ADEM.

A follow-up brain MRI on day 20 of hospitalization showed an almost complete resolution of the previously described signal abnormalities with a residual 3-mm right pons hyperintense T2 FLAIR signal without evidence of diffusion restriction or abnormal enhancement. There was no evidence of newly appearing lesions.

He was discharged home at day 30 with completely normal neurological exam. A brain MRI done 1 month after discharge showed complete resolution of the previously described signal abnormalities with no evidence of abnormal post-contrast enhancement.

A clinical follow-up of the patient over a 12-month period showed a completely normal physical and neurological exam and no episodes of fever or any other complaint. The results of an immunological workup for immune deficiency were normal, including serum immunoglobulin levels, lymphocyte markers, blastogenic response, complement deficiency, oxidative burst and polymorphonuclear chemotaxis. Human immunodeficiency virus (HIV) serology was also negative.

\section{Discussion}

In our case, the patient presented with somnolence, headache, minimal neck stiffness, and high WBC count in CSF. The initial brain MRI showed high suspicion of abscess in the thalami, plus other areas of hyperintense signals on T2 FLAIR at cerebral peduncles, pons and caudate nucleus. The diagnosis of abscess was first considered because of the diffusion restriction and post-contrast annular enhancement as well as the presence of the posterior fossa meningeal enhancement, thus broad spectrum IV antibiotics were given. Risk factors for brain abscess include cardiac diseases, sinus and otogenic infections, poor dental hygiene and complications from dental procedures, infancy, immunosuppression, neurosurgical procedures, penetrating skull injury and comminuted fracture of the skull, congenital lesions of the head and neck (such as dermal sinuses), and a rare complication of meningitis [11]. As in our case, the child presented mild bilateral sinusitis seen on the first MRI and then on the CT scan of the sinus. No immune deficiency has been detected. His brain abscesses could be a complication of the sinusitis or the meningitis although no bacteria were isolated in CSF.

A follow-up brain MRI was done because of the persis- tence of somnolence and showed that the hyperintense lesions on T2 FLAIR were better delineated without newly appearing hyperintense T2 FLAIR lesions and the abscesses have regressed slightly in size and enhancement. ADEM diagnosis was considered to explain the presence of those diffuse demyelinating lesions and the persistent signs of encephalopathy.

ADEM is a rare disorder which most commonly occurs following viral illness usually in the form of a nonspecific upper respiratory infection but may occur after a bacterial or parasitic infection, or after an immunization [8, 12-15] or an administration of drugs. It is an immune-mediated disorder producing multifocal inflammation and subsequent demyelinating lesions within the central nervous system (CNS). The incidence rate is about 8 in 1,000,000. It can occur at any age but is more common in children and adolescents with an average age of 5 to 7 years [14]. ADEM typically begins within 6 days to 6 weeks following an antigenic challenge. The evolution of ADEM may be abrupt, acute, or over a period of few days. Characteristic clinical features include sudden onset of multifocal neurologic disturbances such as visual field defects, aphasia, motor and sensory deficits, ataxia, movement disorders, depressed level of consciousness, focal or generalized seizures and psychosis [16].

ADEM remains a diagnosis of exclusion, always necessitating thorough consideration of alternate diagnoses. The criteria required for the diagnosis of ADEM include a first polyfocal clinical CNS event with presumed inflammatory demyelinating cause, encephalopathy (alteration of consciousness or behavior unexplained by fever, systemic illness, or postictal symptoms), brain MRI abnormalities consistent with demyelination during the acute phase and no new clinical or MRI findings 3 months or more after the clinical onset.

Although the patient responded well to high dose of steroids, the diagnosis of ADEM alone could not be retained and IV antibiotics were given for a total of 4 weeks for suspicion of brain abscesses.

We reviewed the literature to search for a case of ADEM which can present with ring-enhancing and central restriction diffusion lesions on MRI but no such case was found. On the other hand, we tried to search for a case of brain abscesses associated or followed by ADEM and also no such case was described. We know that ADEM typically follows an antigenic challenge, such as infection or vaccination, which activates the immune system [17]. Thus, the most probable hypothesis remains that the brain abscesses triggered an immune reaction and caused an associated ADEM. Another hypothesis would be an ADEM which followed the viral illness that occurred 2 weeks before the admission and all the radiological images would be considered images of ADEM although the central diffusion restriction along with the ring enhancement of the lesion on the initial MRI was not described in any case of ADEM reviewed in the literature.

This case described a challenging diagnosis of ADEM in a patient who initially presented cerebral abscesses on neuroimaging but later turned out to be a case of ADEM associated or maybe caused by the cerebral abscesses. Despite that ADEM is known to follow a febrile infection, a causal relationship has never been established in the literature between ADEM and brain abscesses specifically. Our case could be the first one 
describing ADEM with cerebral abscesses.

\section{Acknowledgments}

None to declare.

\section{Financial Disclosure}

None to declare.

\section{Conflict of Interest}

None to declare.

\section{Informed Consent}

Not applicable.

\section{Author Contributions}

PC and MTB conducted the analysis and wrote the manuscript draft; DE and CG provided access to the data and assistance with study design; PC, DE and PK reviewed the analysis as well as contributed to and edited the manuscript.

\section{References}

1. Mialin R, Koob M, de Seze J, Dietemann JL, Kremer S. Case 173: acute disseminated encephalomyelitis confined to the brainstem. Radiology. 2011;260(3):911-914.

2. Rust RS. Multiple sclerosis, acute disseminated encephalomyelitis, and related conditions. Semin Pediatr Neurol. 2000;7(2):66-90.

3. Tenembaum S, Chitnis T, Ness J, Hahn JS, International Pediatric MSSG. Acute disseminated encephalomyelitis. Neurology. 2007;68(16 Suppl 2):S23-36.

4. Dale RC. Acute disseminated encephalomyelitis. Semin Pediatr Infect Dis. 2003;14(2):90-95.

5. Krupp LB, Tardieu M, Amato MP, Banwell B, Chitnis T,
Dale RC, Ghezzi A, et al. International Pediatric Multiple Sclerosis Study Group criteria for pediatric multiple sclerosis and immune-mediated central nervous system demyelinating disorders: revisions to the 2007 definitions. Mult Scler. 2013;19(10):1261-1267.

6. Alper G. Acute disseminated encephalomyelitis. J Child Neurol. 2012;27(11):1408-1425.

7. Mikaeloff Y, Caridade G, Husson B, Suissa S, Tardieu M, Neuropediatric KSGotFNS. Acute disseminated encephalomyelitis cohort study: prognostic factors for relapse. Eur J Paediatr Neurol. 2007;11(2):90-95.

8. Tenembaum S, Chamoles N, Fejerman N. Acute disseminated encephalomyelitis: a long-term follow-up study of 84 pediatric patients. Neurology. 2002;59(8):1224-1231.

9. Mathisen GE, Johnson JP. Brain abscess. Clin Infect Dis. 1997;25(4):763-779; quiz 780-761.

10. Mueller-Mang C, Castillo M, Mang TG, Cartes-Zumelzu F, Weber M, Thurnher MM. Fungal versus bacterial brain abscesses: is diffusion-weighted MR imaging a useful tool in the differential diagnosis? Neuroradiology. 2007;49(8):651-657.

11. Sheehan JP, Jane JA, Ray DK, Goodkin HP. Brain abscess in children. Neurosurg Focus. 2008;24(6):E6.

12. Zeb Q, Alegria A. Acute disseminated encephalomyelitis (ADEM) following a $\mathrm{H} 3 \mathrm{~N} 3$ parainfluenza virus infection in a pregnant asthmatic woman with respiratory failure. BMJ Case Rep. 2014;2014.

13. Dale RC, de Sousa C, Chong WK, Cox TC, Harding B, Neville BG. Acute disseminated encephalomyelitis, multiphasic disseminated encephalomyelitis and multiple sclerosis in children. Brain. 2000;123(Pt 12):2407-2422.

14. Hynson JL, Kornberg AJ, Coleman LT, Shield L, Harvey AS, Kean MJ. Clinical and neuroradiologic features of acute disseminated encephalomyelitis in children. Neurology. 2001;56(10):1308-1312.

15. Stonehouse M, Gupte G, Wassmer E, Whitehouse WP. Acute disseminated encephalomyelitis: recognition in the hands of general paediatricians. Arch Dis Child. 2003;88(2):122-124.

16. Kumar P, Kumar P, Sabharwal RK. Acute disseminated encephalomyelitis: case report and brief review. J Family Med Prim Care. 2014;3(4):443-445.

17. Pohl D, Alper G, Van Haren K, Kornberg AJ, Lucchinetti CF, Tenembaum S, Belman AL. Acute disseminated encephalomyelitis: Updates on an inflammatory CNS syndrome. Neurology. 2016;87(9 Suppl 2):S38-45. 